\title{
Une expérience pédagogique autour d'un robot industriel
}

\author{
Farid Noureddine \\ Ecole Nationale d'Ingénieurs de Tarbes - ENIT \\ 47, avenue d'Azereix, BP 1629 \\ 65016 Tarbes Cedex \\ Email : farid.noureddine@enit.fr
}

RESUME : Une expérience pédagogique autour d'un robot industriel a été menée à l'Ecole Nationale d'Ingénieurs de Tarbes. En gardant comme objectif des connaissances à acquérir, qui sont celles répertoriées dans le syllabus des enseignements de robotique de l'Enit, une organisation se situant à mi-chemin entre une approche d'enseignement classique et d'enseignement par projet a été adoptée et fait l'objet du présent article.

Mots-clés: Robotique industrielle, CAO Robotique, programmation robot.

\section{Introduction}

L'enseignement de la robotique industrielle, c'est à dire principalement l'étude des bras manipulateurs, est pour beaucoup d'écoles d'ingénieurs partie intégrante du cursus. Les objectifs assignés peuvent sensiblement diverger d'une école à l'autre selon les options considérées et suivant que l'on souhaite former les Elèves-Ingénieurs à l'intégration de robots ou à leur conception.

A l'Ecole Nationale d'Ingénieurs de Tarbes, Enit, l'enseignement est organisé sous forme d'UE : Unités d'Enseignement regroupant des EC, à savoir Eléments Constitutifs. Pour ce qui est de la robotique en Semestre 8 (S8), l'EC '0833GI', dispensé dans l'option Génie industriel traite de l'intégration 
des robots industriels, tandis qu'au semestre S9, une approche davantage orientée sur la conception des robots, voire machines spéciales, est menée dans un autre EC de l'option Contrôle des Processus.

Les détails de ces EC et notamment la répartion horaire entre Cours $(\mathrm{C})$, Travaux Dirigés (TD) et Travaux Pratiques (TP), ainsi que les crédits Ects associés, le programme et le mode d'évaluation retenus sont indiqués en [8].

Il a été décidé de proposer un EC optionnel en S8 centré sur une problématique définie au laboratoire de Robotique de l'Enit. Cette proposition s'adressait aux Elèves-Ingénieurs de l'option Génie Mécanique, donc non inscrits à l'EC '0833GI'. Le challenge était double, d'une part susciter l'intérêt des Elèves-Ingénieurs afin que cet EC puisse ouvrir et d'autre part transmettre les notions fondamentales de robotique. C'est cette expérience qui sera exposée dans cet article, expérience se situant à mi-chemin entre une approche d'enseignement classique et d'enseignement par projet.

\section{Présentation de l'enseignement}

Les différents degrés de liberté de moduler le rythme d'un enseignement, notamment la succession des C, TD et TP dépendent beaucoup de l'effectif. Il est sûr que lorsque l'on dispose d'un effectif inscrit à l'EC inférieur à 12, il peut y avoir une gestion très souple du groupe et, nous le verrons, très réactive de la modulation $\mathrm{C}$, TD et TP. L'expérience menée dans ce travail a donc été pensée en considérant un effectif possible maximum de 12 ElèvesIngénieurs. Le volume horaire attribué à cet EC est de 30 h. réparties en 12 h. de Cours, 10 h. de TD et 8 h. de TP et se déroule sur 8 semaines.

\subsection{Objectifs pédagogiques et motivations}

Tout enseignant se pose tout naturellement et très régulièrement des questions relatives à ses pratiques d'enseignement. Parmi les innovations de ces dernières années, l'enseignement par projet ou par problèmes reste encore une nouveauté qui interpelle les enseignants et les expérimentations menées sont, pour la plupart, globalement positives. Ainsi nous trouvons des initiatives pour des étudiants en Master, [1,5], où est mise en avant l'autonomie de ces derniers. Le développement de leurs compétences en gestion de projet et communication en plus des compétences scientifiques et techniques est recherché dans [6]. Dans le domaine de l'électricité, un approfondissement des 
connaissances est effectué à travers des apprentissages par projet, [2]. Certaines équipes pédagogiques confrontent les étudiants, dès la première année, à la pédagogie par projet comme dans [4] ou à des enseignements de base, par exemple dans le domaine de l'électronique de puissance, [7]. Néanmoins, un certain nombre d'écueils doivent être surmontés pour mener à bien ce type de pédagogie, et notamment la nécessité de fédérer un ensemble d'enseignants, une équipe pédagogique qui arrive à mutualiser un certain nombre d'heures sur une certaine durée, avec un apprentissage transversal des connaissances, comme par exemple en [3]. En fait, ce sont des changements qui impliquent une concertation et des actions mûrement réfléchies et planifiées et qui nécessitent souvent l'implication de la Direction des Etudes. L'expérience décrite ici n'a pas cette envergure puisque c'est la réflexion d'un seul enseignant qui gère son EC et qui peut réagir dans des délais assez courts, vu qu'il ne remet pas en cause le canevas affiché par l'école pour l'EC considéré.

Les circonstances ont joué en notre faveur en ce sens que les conditions optimales pour réaliser une expérience pédagogique profitable à la fois aux Elèves-Ingénieurs pour leurs acquisitions et à l'enseignant pour son expérience, se sont trouvées réunies. Ces conditions sont, d'une part un effectif réduit de 6 étudiants (cet EC est optionnel), d'autre part un volume horaire relativement conséquent et enfin la présence d'un robot récemment acquis et riche en problématiques à résoudre. Il faut noter cependant, que cette ouverture d'EC à effectif réduit n'a été autorisée qu'exceptionnellement du fait du caractère novateur de l'expérience pédagogique, le minimum requis étant normalement de 12 Elèves-Ingénieurs.

La proposition soumise aux Elèves-Ingénieurs a été de définir comme objectif à atteindre à l'issue de ce cours la résolution d'un Cahier Des Charges, CDC, fourni par un client potentiel, ici en l'occurrence le laboratoire de Robotique de l'Enit. L'équipe en charge du projet se composait des 6 ElèvesIngénieurs et de l'Enseignant, la problématique étant clairement définie et précisée plus avant. Les Elèves-Ingénieurs et l'Enseignant ont uni leur efforts pour avancer le plus loin possible dans la résolution du CDC. Différemment de l'approche projet, où les solutions sont cherchées et amenées par les ElèvesIngénieurs, l'Enseignant se trouve disponible, de par ses connaissances, pour pouvoir dispenser du $\mathrm{C} / \mathrm{TD}$ afin de répondre aux interrogations des ElèvesIngénieurs. Les problèmes ont été posés séquentiellement en début de séance et identifiés par les Elèves-Ingénieurs eux-mêmes, des propositions de solutions ont alors été discutées. L'Enseignant avait donc un rôle d'animateur et répondait aux interrogations soulevées par chaque binôme, par le biais de 
$\mathrm{C} / \mathrm{TD}$ dispensés à l'ensemble des Elèves-Ingénieurs.

En fait, le CDC défini au Laboratoire de robotique a été pensé pour susciter des interrogations et aboutir à l'apprentissage de deux importantes parties des connaissances répertoriées dans le syllabus des enseignements de robotique de l'Enit, à savoir :

- l'intégration des robots industriels,

- la modélisation géométrique de ces derniers.

\section{Présentation du travail - Organisation des tâches}

Du fait de l'acquisition récente par le laboratoire de Robotique d'un nouveau robot (Kuka, 6 axes), montré en figure (1), nous nous sommes fixé comme objectif la mise en exploitation de ce robot et la définition d'une cellule associée. Ce projet a été divisé en 3 sous projets affectés à chacun des 3 binômes d'Elèves-Ingénieurs.

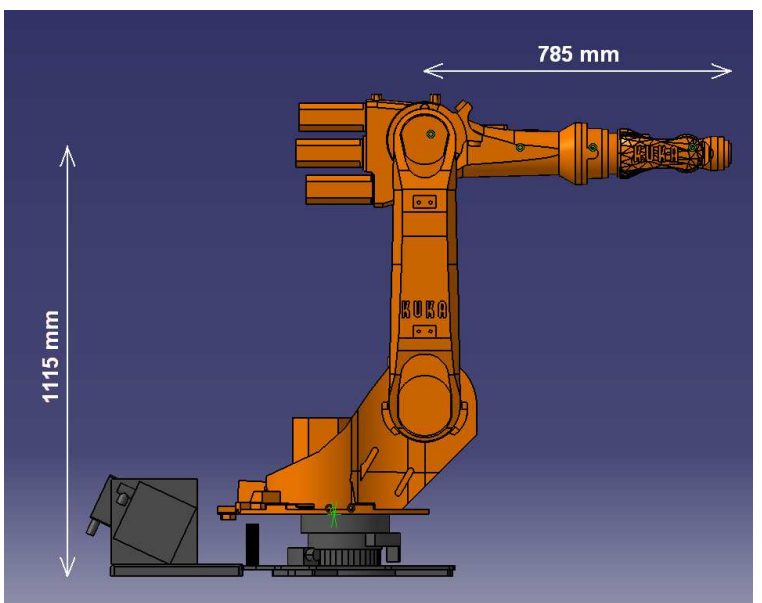

Figure 1: Entité robot créée sous Delmia

Le laboratoire de Robotique de l'Enit dispose de KukaSimPro et Officelite, produits de la société Kuka $\mathrm{GmbH}$, logiciels de CAO pour les robots Kuka et de Delmia, produit de la société Dassault Systèmes, logiciel de CAO pour la conception de cellules robotiques. Cet ensemble a permis de définir 
et de proposer des tâches diverses aux Elèves-Ingénieurs pour les trois sous projets. Le groupe 1 a ainsi travaillé entièrement sur de la simulation, le groupe 2 a officié sur le robot et le groupe 3 a réalisé la programmation hors ligne avec validation de ses programmes sur le robot.

1. Groupe Delmia : Conception de la cellule robotique sous Delmia Prise en main de Delmia Création du modèle $\mathrm{CAO}$ du robot

2. Groupe Kuka : Programmation et mise en sécurité du robot Prise en main du robot Etude des interfaces Entrées/Sorties

3. Groupe KukaSim : Programmation de la cellule robotique sous KukaSim

Création du modèle CAO de la cellule

Programmation hors ligne du robot

Validation des programmes sur le robot

Tels que présentés, il est important de définir sur les 3 sous projets une tâche de fond qui soit active dès la première séance afin d'éviter tout temps mort qui survient fréquemment lors des démarrages de projets. Des séances de $4 \mathrm{~h}$ ont été programmées, permettant ainsi un début de séance en autonomie, puis une évolution de la séance vers du Cours ou du TD suivant les interrogations soulevées par les Elèves-Ingénieurs. Les problèmes posés par les 3 groupes sont hiérarchisés en terme d'urgence pour lever les points de blocage et une programmation de $\mathrm{C} / \mathrm{TD}$, pour un point donné, est définie pour l'ensemble des Elèves-Ingénieurs. Chacun bénéficie ainsi de la mise en évidence des problèmes et surtout de leur résolution. Les résultats des 3 groupes sont maintenant présentés dans les sections suivantes.

\section{Groupe Delmia - Conception de la cellule robotique sous Delmia}

La version du logiciel Delmia est antérieure à l'achat du robot et de ce fait, ce dernier ne figure pas dans la bibliothèque des robots. Cela a conduit, du point de vue pédagogique, à des problèmes intéressants à identifier par les Elèves-Ingénieurs et naturellement à résoudre. 
Delmia est un logiciel qui permet la conception de cellules robotisées. La visualisation et validation de toutes les séquences sont réalisées hors ligne puis téléchargées sur l'armoire de commande pour exploitation de la cellule. Comme c'est souvent le cas pour tous les gros logiciels, l'apprentissage s'avère relativement long et une exploitation optimale des fonctions nombreuses et variées n'interviendrait que si les Elèves-Ingénieurs utilisaient quotidiennement ce logiciel dans leur travail, ce qui ne peut naturellement pas être le cas. Néanmoins, le travail dans le cadre de ce projet a permis aux Elèves-Ingénieurs de bien appréhender les possibilités de ce logiciel et de disposer des clés pour en approfondir l'apprentissage.

\subsection{Objectif}

L'apprentissage de Delmia est donc posé comme tâche de fond, le but fixé étant le dessin de la cellule définie au laboratoire d'Electronique-Vision. Ainsi, les Elèves-Ingénieurs sont immédiatement opérationnels et également à l'écoute du groupe Kuka pour des sollicitations, notamment dans la maîtrise du positionnement des éléments de la cellule.

Il leur a également été demandé de réfléchir au point clé suivant :

- Le robot KR6arc ne figure pas dans la bibliothèque de Delmia, comment alors procéder pour ajouter un élément dans cette bibliothèque ? Cela concerne, dans notre cas, le modèle d'un robot mais cela aurait pu tout aussi bien concerner le modèle d'une machine spéciale conçue par rapport à un $\mathrm{CDC}$ et ne disposant pas de modèle CAO.

\subsection{Déroulement du projet}

Les étapes réalisées par ce groupe ont été et ce, afin de se familiariser avec Delmia, de dessiner les éléments de la cellule réelle telle qu'elle se présente au laboratoire, voir figure (2). Il est à noter que les Elèves-Ingénieurs de l'Enit ont une formation en conception mécanique sous Catia, logiciel de CAO mécanique également développé par Dassault Systèmes. L'environnement et les formalismes sont assez identiques, ce qui a naturellement facilité l'apprentissage de Delmia.

Pour créer le modèle CAO du robot, les Elèves-Ingénieurs ont dû :

- S'enquérir de l'existant et récupérer auprès du constructeur Kuka le dessin (géométrie) du robot KR6arc. Un fichier au format d'échange 
STEP a ainsi été reçu.

- S'appuyer sur leur connaissance de Catia pour créer les entités exportables vers Delmia. Les différents éléments de chaque bras ont été regroupés sous un élément, les liaisons rotoïdes ont ensuite été définies et l'entité robot définie et exportée sous Delmia. Les animations par axes ont ainsi pu être menées.

A cette étape du projet, il a été demandé aux Elèves-Ingénieurs de réfléchir à la possibilité de déplacer les axes du robot en mode cartésien dans le repère Robot par exemple, c'est à dire le repère cartésien attaché au socle du robot. Une sollicitation des 3 groupes a permis d'engager une discussion sur les implications algorithmiques que devait posséder le générateur de trajectoires pour réaliser ce déplacement. Nous sommes bien sûr arrivés à expliciter ce que l'on appelle en robotique le modèle géométrique inverse et ainsi un $\mathrm{C} / \mathrm{TD}$ a été abordé afin d'apprendre la résolution d'un modèle géométrique inverse dans le cas de robots à chaîne cinématique simple. Un cours classique avec présentation des matrices homogènes et utilisation de la méthode de DenavitHartenberg a ainsi été dispensé, (la méthode de Denavit-Hartenberg est une méthode assez répandue pour l'enseignement des modèles des robots industriels), par contre, seul le robot concerné a été étudié. Là où une approche complète avec étude de 2 ou 3 exemples illustratifs des architectures de robots les plus courantes aurait nécessité une douzaine d'heures de C/TD, nous y avons consacré à peu près la moitié.

A l'issue de cette étude, le groupe disposait du modèle géométrique inverse. La procédure de couplage de ce modèle dans l'environnement Delmia n'a cependant pu être menée à terme du fait de la densité des échanges réalisés avec nos interlocuteurs "Delmia" et des contraintes de temps.

Un dessin de la cellule du laboratoire a enfin été réalisé afin de réfléchir aux implantations futures d'un environnement périrobotique, et notamment l'implantation d'un miniconvoyeur. Les outils utilisés, tels la gestion de l'espace de travail 3D, se sont révélés efficaces et ont permis d'initier des échanges avec les autres groupes puisque cette demande d'information émanait du groupe Kuka en charge du travail sur le robot réel. 


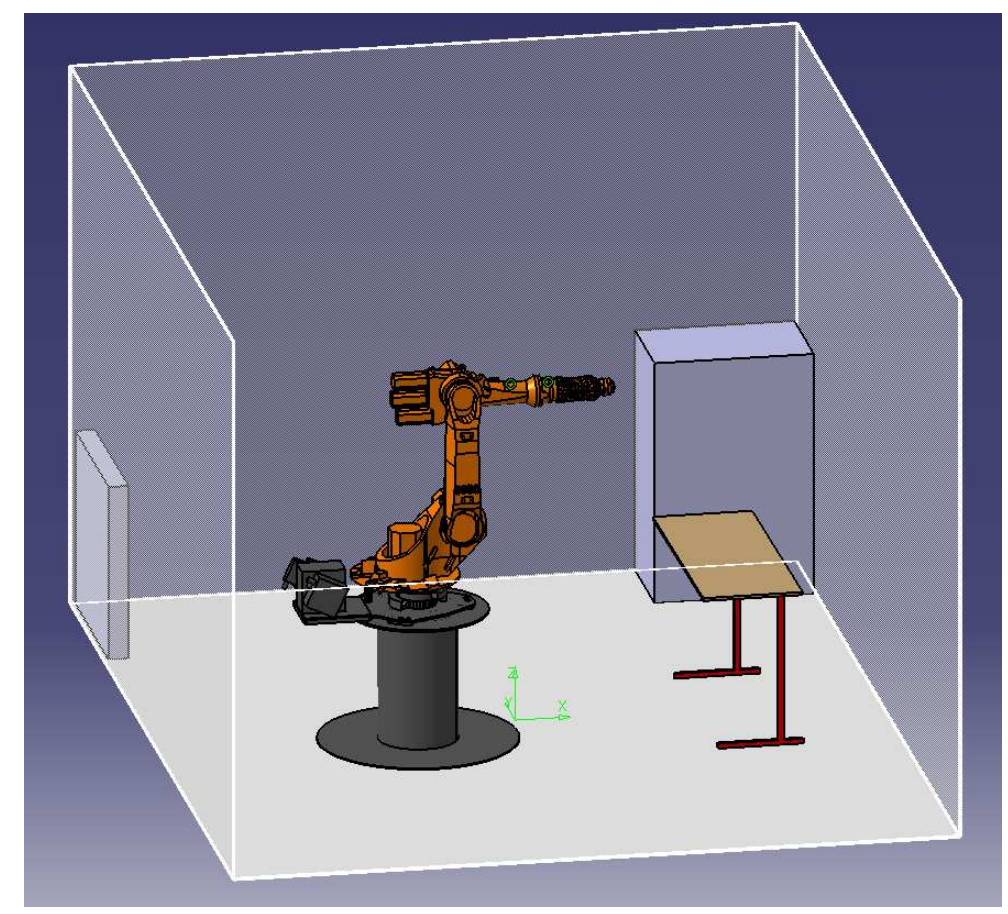

Figure 2: Cellule robotique

\section{Groupe Kuka-Programmation et mise en sécu- rité du robot}

Le robot Kuka Kr6arc que nous avons monté sur un socle, voir figure (3) est un robot anthropomorphe 6 axes initalement dédié au soudage à l'arc, mais qui, de par ses performances globales, soit une répétabilité de l'ordre du 1/10ème de mm, et une charge maximale autorisée égale à $6 \mathrm{~kg}$, peut très bien convenir à bien d'autres tâches à effectuer dans les ateliers de production. L'attrait de travailler sur un robot industriel est certain pour nos ElèvesIngénieurs, même si la vigilance doit être de rigueur du fait des consignes de sécurité à respecter. 


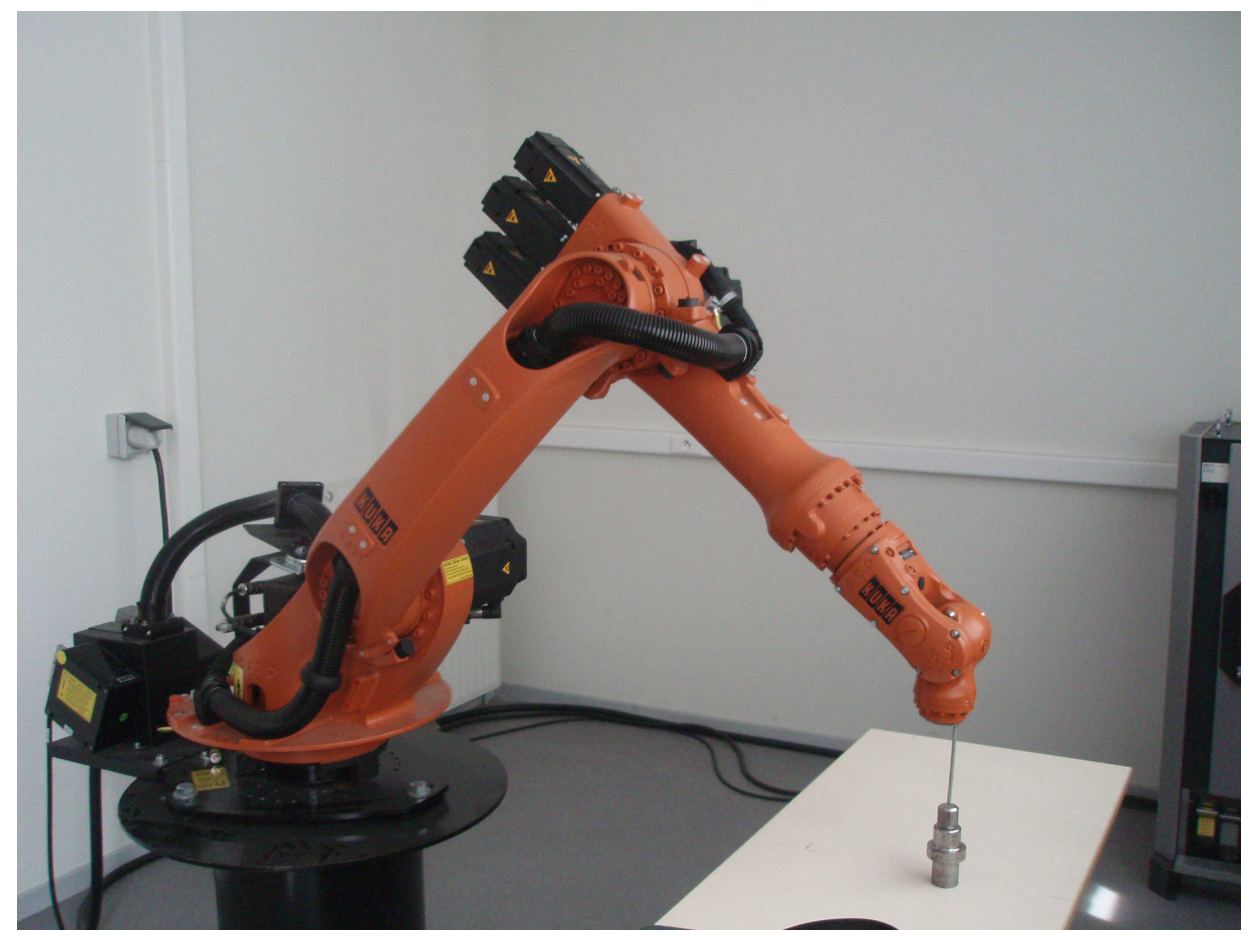

Figure 3: Robot anthropomorphe Kuka, KR6 Arc

\section{$5.1 \quad$ Objectifs}

La tâche de fond affectée à ce groupe était tout indiquée et concernait la programmation du robot. Différents scénarios ont été définis et des programmes écrits et validés sur le robot, mettant en jeu :

- la programmation axe par axe et en cartésien sur l'ensemble de l'espace de travail dans les repères base, robot et outil

- La commande du robot par la programmation de variables booléennes

- L'interruption du cycle du robot par les entrées analogiques

En point de réflexion, ce groupe devait travailler sur la mise en sécurité du robot par la commande d'un portillon muni d'un interrupteur de sécurité. 


\subsection{Déroulement du projet}

En s'appuyant sur la documention fournie par le constructeur, la programmation du robot sous ses différents aspects a pu être menée sans difficulté majeure. La sollicitation des Elèves-Ingénieurs est intervenue à partir du moment où la communication hardware avec le robot a dû être activée, ainsi des éléments de cours concernant :

- la gestion des modules d'E/S à ajouter dans l'armoire de commande du robot ainsi que

- la gestion des E/S liées aux différents capteurs et actionneurs généralement disponibles dans une cellule robotisée

ont été rappelés. Il faut souligner que les étudiants de l'Enit ont préalablement suivi, au cours de leur scolarité, des cours d'automatisme et que de ce fait, ce travail a permis de fixer les concepts déjà abordés par une utilisation concrète de leurs connaissances.

Concernant la mise en sécurité, il a été décidé de limiter la zone d'accès au robot, voir figure (4), par une protection passive. La porte (ouverture vers l'extérieur, passage de $80 \mathrm{~cm}$ ) est équipée d'un interrupteur de sécurité connecté au robot.

Les plans et devis ont été réalisés et la fabrication est en attente de lancement par le CIMMES (Centre d'Ingéniérie en Mécanique, Matériaux et Surfaces) de l'Enit.

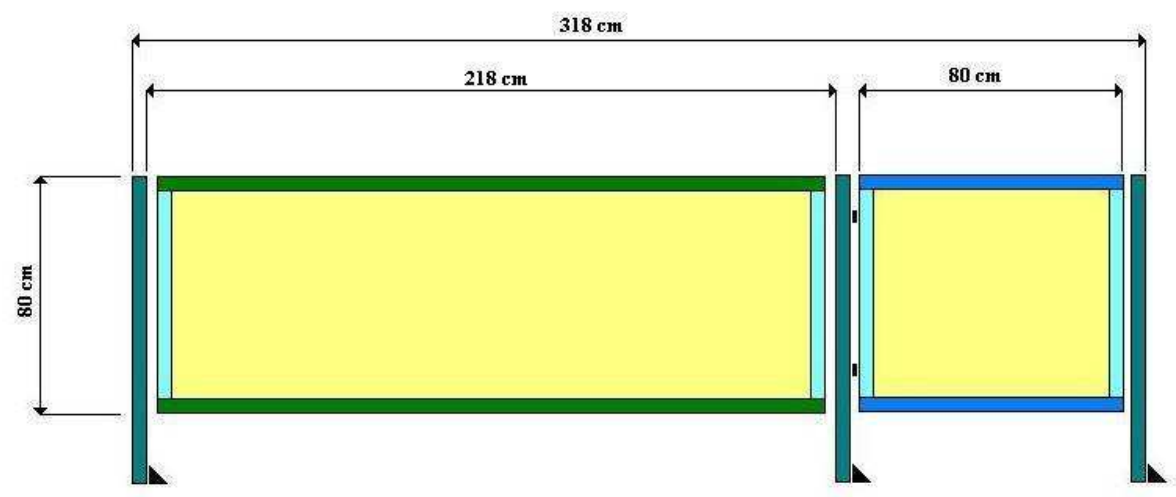

Figure 4: Dispositif de sécurité 


\section{Groupe SimPro-Programmation hors-ligne du robot Kuka KR6 Arc}

La version Ecole du package de simulation KUKA regroupe un ensemble de logiciels permettant la création et visualisation en 3D des cellules avec des robots de la gamme Kuka, l'écriture et l'optimisation des programmes hors ligne, l'analyse des temps de cycles et enfin le téléchargement et l'exécution sur le robot réel des programmes conçus hors ligne.

\section{$6.1 \quad$ Objectifs}

L'objectif principal assigné à ce groupe a été la réalisation de programmes hors ligne et la validation de ces programmes sur le robot. En parallèle, les Elèves-Ingénieurs devaient apprendre à créer et simuler des trajectoires.

\subsection{Déroulement du projet}

La cellule du laboratoire a ainsi été dessinée, comme montré en figure (5) où les 3 murs ont été remplacés par 3 panneaux et nombre de fonctionnalités du logiciel SimLayout ont ainsi pu être explorées. Les Elèves-Ingénieurs étaient la plupart du temps pratiquement autonomes, et ce groupe s'est révélé être, sans nul doute, celui qui a soulevé le moins de questions en rapport à l'optique de déclencher, de la part de l'Enseignant, des séquences de cours/TD.

En fait, les questions qui se posaient étaient toujours relatives à l'utilisation du logiciel et souvent très pointues et spécifiques et ont plutôt nécessité un contact avec le support technique de Kuka. 


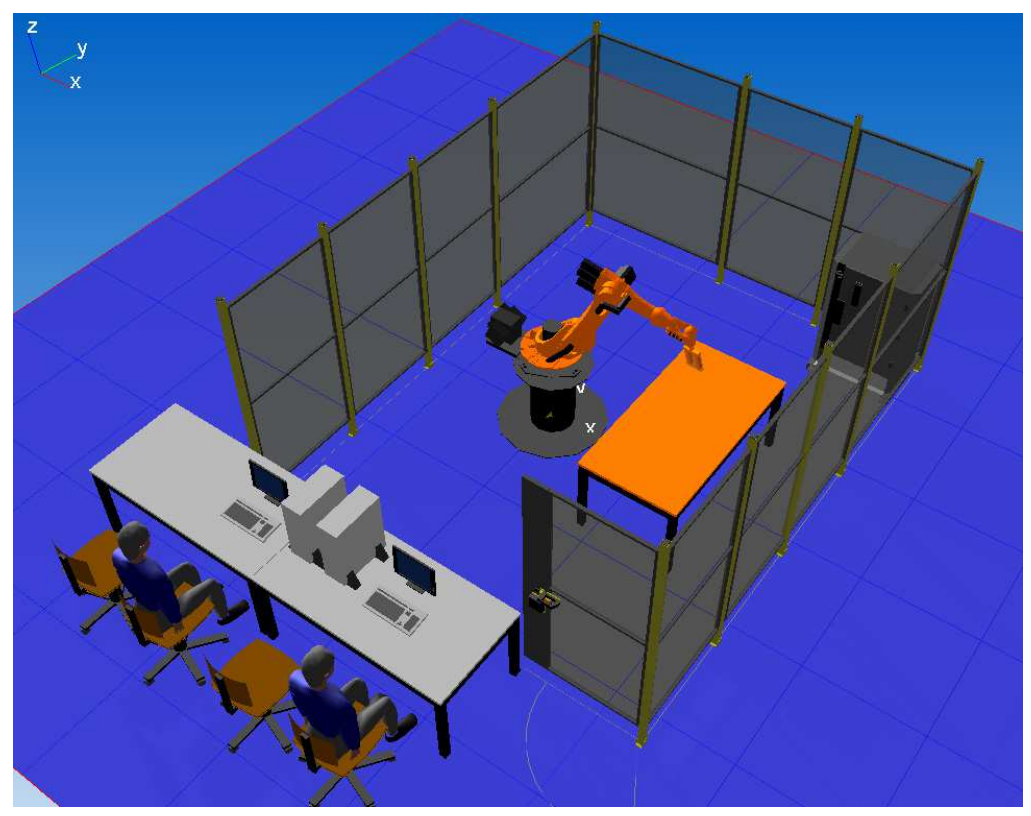

Figure 5: Cellule modélisée sous KukaSim

L'objectif a été atteint en fin de stage et le téléchargement de programmes hors ligne réalisé via le réseau ou par clé USB sur l'armoire de commande. Très peu d'exploitations ont été menées pour pouvoir apprécier la qualité de cette programmation hors ligne, mais c'est désormais un outil exploitable et qui pourra amener des réponses pratiques quant aux performances de ce type de programmation en comparaison avec la programmation par apprentissage.

\section{Bilan et Conclusion}

Les Elèves-Ingénieurs ont été sollicités pour participer à une évaluation de cet enseignement à travers le renseignement d'un questionnaire. Ce dernier permet d'enrichir les conclusions données à la fin de ce paragraphe.

\subsection{Questionnaire - Propositions d'amélioration des Elèves- Ingénieurs}

Ce questionnaire d'évaluation a été distribué et renseigné de façon anonyme au terme de l'enseignement. 
Les réponses pouvaient être de la forme suivante : Oui, Plutôt Oui, Non, Plutôt Non, Sans Avis

1. La densité des connaissances acquises vous semble t-elle suffisante?

2. L'organisation de points de cours suite à une problématique apparaissant lors du déroulement du projet vous paraît-elle plus motivante?

3. Le passage, au cours d'une même séance de $4 \mathrm{~h}$, d'une phase de travail en autonomie à une séance de cours n'est pas souhaitable?

4. La part du travail en autonomie devrait-elle être moins importante par rapport à la partie Cours?

5. Le traitement des points théoriques (Etudier, par exemple, d'autres modèles de robots) devrait-il être plus approfondi (faire plus de TD) ?

6. Le travail en autonomie devrait-il être plus guidé ?

7. Ce type d'enseignement vous paraît-il intéressant, même si votre charge de travail ne vous permet pas de consacrer un travail supplémentaire hors séance?

8. Les conditions matérielles ont elles été satisfaisantes ?

9. L'organisation de l'évaluation : rapport + soutenance, vous semble t-elle pertinente?

10. Si vous aviez le choix, reprendriez vous cet enseignement?

- Avez vous des propositions pour améliorer cet enseignement?

\subsubsection{Réponses aux questions}

Dans le tableau ci-dessous est noté le nombre d'Elèves-Ingénieurs ayant répondu à la question concernée. 


\begin{tabular}{|c|c|c|c|c|c|}
\hline & Oui & Plutôt Oui & Non & plutôt Non & Sans Avis \\
\hline \hline 1 & 2 & 3 & & 1 & \\
\hline 2 & 1 & 5 & & & \\
\hline 3 & & 2 & & 3 & 1 \\
\hline 4 & & & 4 & 2 & \\
\hline 5 & & & & 6 & \\
\hline 6 & 1 & 4 & & 1 & \\
\hline 7 & 5 & 1 & & & \\
\hline 8 & 6 & & & & \\
\hline 9 & 4 & 2 & & & \\
\hline 10 & 5 & 1 & & & \\
\hline
\end{tabular}

\subsubsection{Propositions d'amélioration des Elèves-Ingénieurs}

Les propositions d'amélioration des Elèves-Ingénieurs sont listées ci-après :

- Le cahier des charges doit être plus précis

- Une plus grande interaction entre les binômes

- Des séances plus rapprochées dans le temps

- Des objectifs par séance

- Des permutations sur les postes de travail

\subsection{Discussion et Conclusion}

Une analyse des réponses au questionnaire décrit au paragraphe 7.1 permet de tirer un certain nombre d'enseignements. Cette approche pédagogique est plutôt plébiscitée par rapport à une approche classique (Questions 7 et 5). Toutefois, cet enthousiasme peut être nuancé par le fait que (question 6), les Elèves-Ingénieurs sont aussi demandeurs de plus de points de repères.

Les propositions d'améliorations sont aussi quelque peu contradictoires, avec cet enthousiasme déclaré, puisque il est souhaité un cahier des charges plus précis et des objectifs par séances. En fait, les Elèves-Ingénieurs sont demandeurs de points de repères pour, en quelque sorte s'autoévaluer en fin de séance et se rassurer sur les résultats obtenus et le travail fourni.

En ce qui concerne l'interaction entre groupes, elle a été suscitée, par exemple lorsque le groupe $\mathrm{CAO}$ devait télécharger ses programmes sur le 
robot, mais cette interaction est restée, il est vrai, trop souvent occasionnelle. C'est un point qui demande un travail bien spécifique de définition des thèmes des groupes et qui sans aucun doute est un point à améliorer pour la réussite de futures expériences pédagogiques.

Tous les thèmes n'ont pas suscité le même intérêt et cette expérience pédagogique basée sur le couplage entre mise en évidence d'un problème et réaction de la part de l'enseignant sur du Cours/TD a excellement fonctionné sur le groupe Delmia, bien sur le groupe Kuka et de manière moins satisfaisante sur le groupe KukaSim.

Il est manifeste que le thème du groupe Delmia a été pensé de telle façon que les Elèves-Ingénieurs arrivent à un moment en butée sur le Modèle Géométrique Inverse (MGI) afin que ce dernier puisse leur être enseigné. Cette voie royale pour justifier un enseignement, en l'occurence l'étude du MGI, ne s'est pas retrouvée de façon aussi flagrante dans les thèmes des autres groupes. Cet enseignement se trouve t-il de ce fait être mieux assimilé ? D'après les réponses aux questionnaires d'évaluation des Elèves-Ingénieurs, présentées en section précédente et sans fausse modestie, on serait enclin à répondre par l'affirmative.

Le manque de recul sur cette expérience nous interdit pour l'instant d'affirmer une préférence ou même de pouvoir établir une comparaison entre les Elèves-Ingénieurs ayant suivi le cursus classique de l'enseignement de la robotique et ceux concernés par cet EC expérimental. En l'état de nos observations, il semble qu'en terme d'exhaustivité d'acquisition d'un ensemble de connaissances brutes, de cohérence d'apprentissage dans le temps, un enseignement classique qui déroule une forme standard $\mathrm{C} / \mathrm{TD}$ et $\mathrm{TP}$ se révèlera plus approprié. Cependant, La continuité de ce type d'expérience pourra permettre de dégager des voies de réflexion pertinente pour une complémentarité de ces deux modes d'apprentissage.

Si, du point de vue de l'Enseignant, cette expérience a été au moins aussi enrichissante que pour les Elèves-Ingénieurs, elle s'avère être sûrement moins confortable que de l'enseignement standard du fait de sollicitations sur des problèmes auxquels les réponses ne sont toujours pas évidentes. L'expérience de l'Enseignant est sans aucun doute un point important pour mener à bien ce type de travail. Il s'agit non seulement de faire face aux problèmes scientifiques et techniques mais également de faire en sorte que la motivation de départ des Elèves-Ingénieurs, qui ont choisi en toute connaissance de cause d'intégrer cet EC dans leur cursus, ne s'amenuise pas, mais au contraire se renforce tout au long du semestre. 


\section{Remerciements}

Je remercie Emmanuel, Florian, Romain, Sébastien, Simon et Vincent de la $44^{\text {ème }}$ promotion de l'Enit pour leur investissement dans ce projet. Leur travail et leur implication au laboratoire, voir figure([6), auront permis une amélioration des outils dorénavant disponibles.

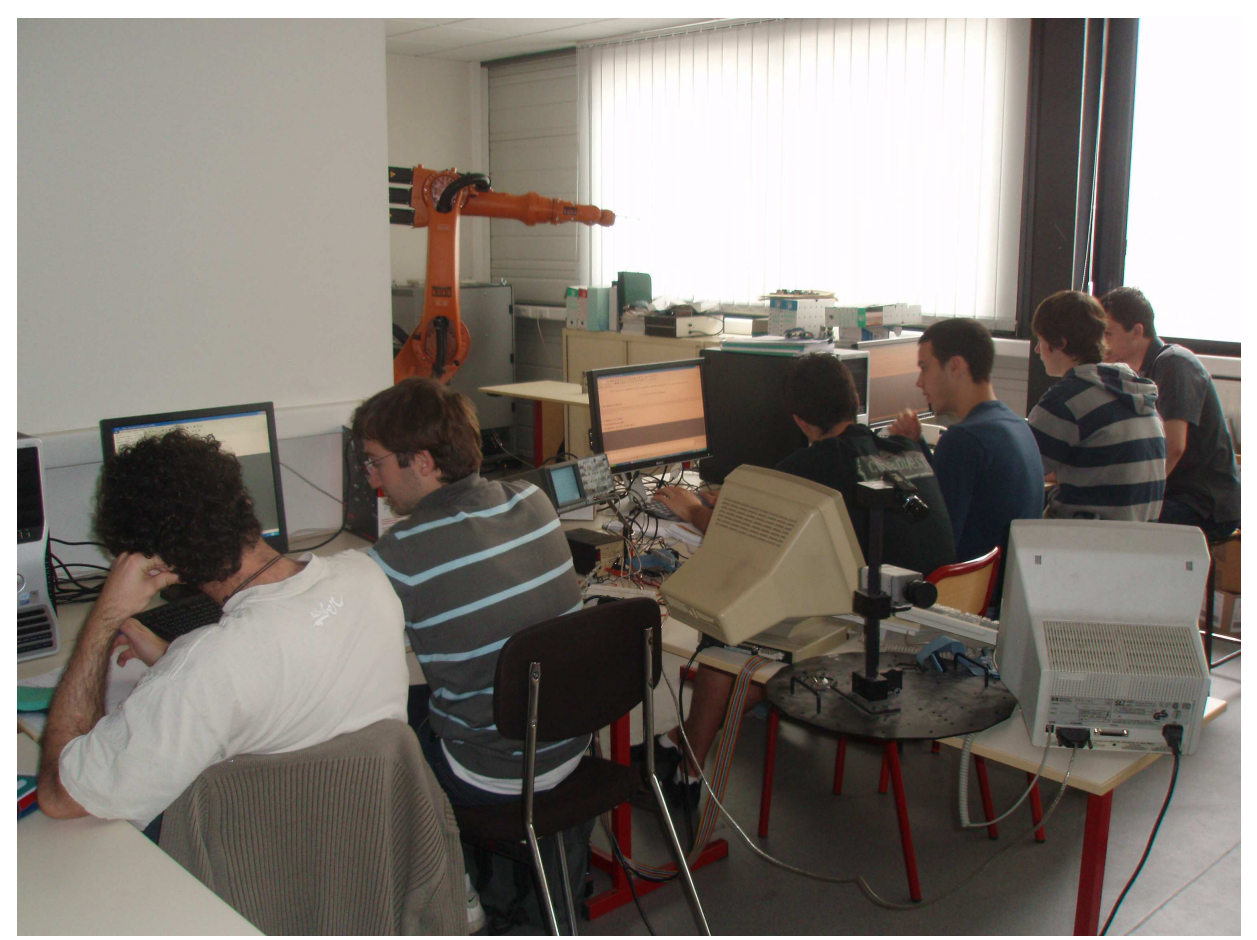

Figure 6:

Je remercie également José Guimarès, Technicien au laboratoire de Robotique, pour sa disponibilité permanente et son aide au bon déroulement de ce projet.

\section{References}

[1] A. Nonclercq, K. De Cuyper, E. Leroy, D. Lopez Martinez, F. Robert, Apprentissage par projet : L'utilisation conjointe de chaînes 
d'acquisition réelle et virtuelle pour enseigner l'instrumentation. Journal sur l'Enseignement des Sciences et Technologies de l'Information et des Systèmes, J3eA 8 HORS SÉRIE 1 (2009) 1005, DOI: 10.1051/j3ea:2008046.

[2] L. De Vroey, F. Vrins, F. Labrique, C. Trullemans, C. Eugène, D. Grenier, Apprentissage par projet en électricité : exemple et mise en oeuvre. Je3A 5 (2006), DOI: 10.1051/j3ea:2006020.

[3] A. Tauvel, N. Simond, S. Todeschini, Utilisation de la simulation dans des projets de robotique. J3eA 8 HORS SÉRIE 1 (2009) 1018 , DOI: 10.1051/j3ea:2008059.

[4] M. Blondeau, A. De Greef, P. A. Douxchamps, B. Genêt, M. Haelterman, C. Lenders, E. Leroy, P. Nardone, V. Raman, A. Richard, F. Robert, Apprentissage par projet : Réalisation d'une éolienne urbaine en matériaux de récupération. J3eA 8 HORS SÉRIE 1 (2009) 1028, DOI: $10.1051 / \mathrm{j} 3 \mathrm{ea} / 2009002$.

[5] L. Baghli, A. Rezzoug, Lévitation magnétique, une approche objet-projet. J3eA 9 (2010) 0002, DOI: 10.1051/j3ea/2010002.

[6] D. Dalle, P. Mabilleau, N. Boutin, G. Lachiver, L'apprentissage par problèmes et par projets pour le développement des compétences professionnelles de l'ingénieur : l'exemple des programmes de génie électrique et de génie informatique de l'Université de Sherbrooke, J3eA 7 HORS SÉRIE 1 (2008) 1029, DOI: 10.1051/j3ea:2008028.

[7] F. Plumier, P. Bleus, C. Geuzaine, Collaboration Industrielle et Enseignement par Projet en Electronique de Puissance. J3eA 8 HORS SÉRIE 1 (2009) 1015, DOI: 10.1051/j3ea:2008056..

[8] http://www.enit.fr/sr/239/index.php 